\title{
Trapping and detrapping effects in high-quality chemical-vapor-deposition diamond films: Pulse shape analysis of diamond particle detectors
}

\author{
Marco Marinelli, E. Milani, A. Paoletti, A. Tucciarone, and G. Verona-Rinati \\ INFM-Dipartimento di Scienze e Tecnologie Fisiche ed Energetiche, Università di Roma "Tor Vergata," Via di Tor Vergata, \\ I-00133 Roma, Italy \\ M. Angelone and M. Pillon \\ Associazione EURATOM-ENEA sulla Fusione, Via E. Fermi 27, I-00044 Frascati (Roma), Italy \\ (Received 12 February 2001; published 19 October 2001)
}

\begin{abstract}
An analysis of the time evolution of the response of diamond particle detectors is carried out, using as a probe $5.5 \mathrm{MeV} \alpha$ particles impinging on high-quality diamond films grown by microwave chemical vapor deposition (CVD). Both the amplitude and the time evolution of the pulses are shown to change drastically when the detector is preirradiated with $\beta$ particles (pumping), a slow component developing after pumping, indicating carriers trapping and releasing (detrapping). Pulse shapes obtained for positive and negative detector polarities are compared in both the as-grown and pumped states. The presence of at least two trapping centers for holes is necessary to explain the results, the shallower having an activation energy of about $0.3 \mathrm{eV}$. The effects of pumping are clarified, and the different role played by electrons and holes is evidenced. We modify a previous model for trapping-detrapping behavior originally applied to $\mathrm{Si}(\mathrm{Li})$ detectors to describe the more complex behavior of CVD diamond detectors, and develop a computer simulation based on it. The simulated pulse shapes agree very well with experiment with reasonable values of the physical parameters involved, making this technique helpful for studying and identifying defects which are responsible for limitation of the efficiency of CVD diamond particle detectors. Field-assisted detrapping seems to take place for fields of about $10^{4} \mathrm{~V} / \mathrm{cm}$.
\end{abstract}

DOI: 10.1103/PhysRevB.64.195205 PACS number(s): 61.82.Fk, 61.72.-y, 81.05.Tp, 29.40.Wk

\section{INTRODUCTION}

With the next generation particle accelerators, the conventional silicon-based particle detectors will not be able to sustain the high projected particle fluxes without rapid deterioration. Possible alternatives are "hardening" treatments of silicon or the use of new materials, among which diamond shows extremely high radiation hardness. ${ }^{1}$ In addition, diamond presents interesting electronic properties, ${ }^{2}$ such as high carrier mobility, a high energy gap resulting in a very low number of free carriers, and a very high breakdown field, thus in principle making low noise, fast diamond based detectors feasible.

Because of its cost, moderate size, and lack of standardization natural diamond is not suitable for such an application. High-pressure synthesized diamond detectors with excellent properties have been realized ${ }^{3}$ but mainly because of size problems most of the research in this field is focused on nuclear detectors based on synthetic diamond films grown by chemical vapor deposition (CVD), whose performances are studied in many research institutes, commercial diamond producers, and particle accelerators plants (see, e.g., the RD42 activity at CERN). ${ }^{1}$

Efficient particle detection requires high drift lengths of the carriers produced by the ionizing particle and is therefore highly sensitive to film quality. Therefore a severe limitation for this as well as for many other applications of diamond is the presence of defects and the polycrystalline nature of CVD diamond films. It is important both to realize high quality diamond films and to understand the role of defects and grain boundaries in the detection mechanism. Due to the complexity of CVD diamond, very little is known nowadays on this subject.

An interesting feature is the priming (or pumping) effect, which is widely used to increase CVD diamond detector performance. It consists of a preirradiation of the CVD diamond detector by ionizing radiation. The increased efficiency is believed to be due to a saturation of deep traps. In spite of the importance of this effect, only a few authors studied the relationship between pumping and the microscopic structure of CVD diamond. ${ }^{4-6}$

The performance of diamond particle detectors and the effects of pumping are generally described through the charge collection distance and the efficiency, which we define below. In a standard experimental setup in which a parallel plate detector of thickness (i.e., electrode spacing) $d$ is used, each electron-hole pair created by an ionizing particle induces ${ }^{7,8}$ in the external circuit a charge $q_{c}=e x / d, x$ being the total distance that the electron and hole move apart under the action of the applied electric field before either reaching the electrode or being trapped, and $e$ being the electronic charge. The amplitude of the voltage pulse measured by the external circuit is proportional to the total collected charge $Q_{c}$ (i.e., the sum of $q_{c}$ over all electron-hole pairs produced by the ionizing particle) and the time evolution of the pulse depends on carriers' dynamics. The ratio

$$
\eta=Q_{c} / Q_{0}
$$

between $Q_{c}$ and the total charge $Q_{0}$ generated by the ionizing particle in the detector is the detector's efficiency. Clearly, $\eta$ depends on the average drift distance 


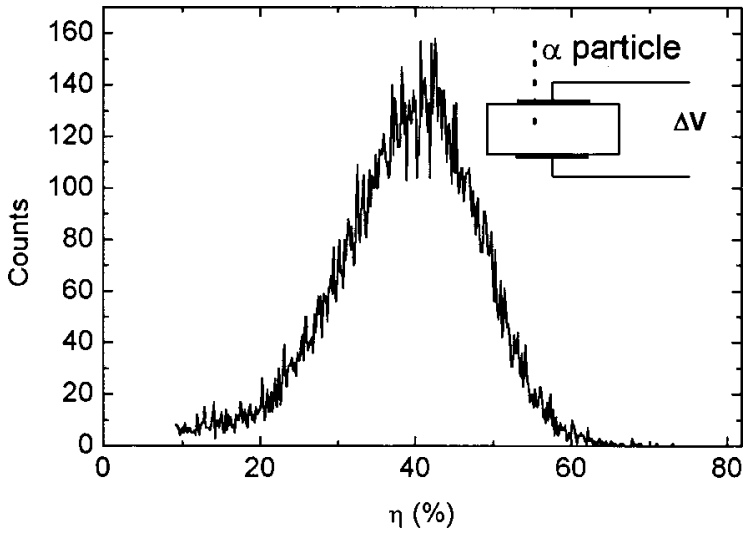

FIG. 1. Typical $\alpha$-particle collection spectrum of our films in the pumped state. Inset: geometry of the parallel-plate detector.

$$
\delta=\left(\mu_{e}+\mu_{h}\right) \tau E,
$$

where $\mu_{e}, \mu_{h}$ are the electron and hole mobilities, respectively, $\tau$ is the mobility weighted lifetime of electrons and holes, and $E$ is the applied electric field. The average drift distance $\delta$ is also referred to as the charge collection distance (CCD).

The statistical distribution of the amplitudes of the pulses generated by the incoming radiation (i.e., the charge collection spectrum; see Fig. 1 for a typical spectrum of our detectors in the pumped state) is the preferred tool to analyze the behavior of particle detectors, showing both the amplitude of the response to particle irradiation (that is, the induced charge and therefore the detector's efficiency and CCD) and the inhomogeneity of the response (that is the energy resolution). However, apart from these performance parameters, limited information on the physical process leading to particle detection can be obtained from the charge collection spectrum. Recently ${ }^{4}$ a model discussing the role of in-grain defects and grain boundaries in determining the response of detector grade CVD diamond films has been proposed which relates the change in the shape of the charge collection spectrum before and after pumping to the distribution of defects. Soon after, ${ }^{5}$ this model was found to be able to quantitatively explain the dependence of efficiency and CCD on the thickness of CVD diamond detectors in the as-grown and pumped states, taking into account the polycrystalline nature of CVD diamond and its columnar growth.

An interesting technique which has been used by several authors to collect information on the efficiency and CCD of diamond samples is ion beam induced charge (IBIC). In IBIC a beam of ions (typically protons) is focused in a micrometer-size spot on the surface of a diamond detector, so that information on the local efficiency and CCD of the sample can be extracted. The detector area is then scanned to obtain a two-dimensional (2D) map of the geometrical distribution of efficiency and CCD over the detector's area. With this technique it has been shown that the response of CVD diamond detectors is inhomogeneous ${ }^{9,10}$ and that pumping increases the efficiency of "bad" regions. ${ }^{6} \mathrm{Al}-$ though very powerful, thanks to its spatial resolution, the IBIC technique provides limited information concerning carrier dynamics.
A deep insight on the dynamics of charges generated in the detector can be obtained from the time development of single pulses. Zanio et al. ${ }^{11}$ and Martini and McMath ${ }^{12}$ showed how this time behavior changes when detrapping and/or trapping events occur in the detector, and applied this analysis to conventional $\mathrm{Si}$ detectors. In this paper we present the time evolution of charge pulses in detector grade CVD diamond films and, by extending the approach of Martini and McMath to describe the more complex behavior of CVD diamond detectors, succeeded in describing the trapping-detrapping processes taking place in CVD diamond, uncovering the different role played by electrons and holes. The effects of pumping are especially investigated. A computer simulation based on this model is shown to agree well with the experimental data, thus allowing the extraction of quantitative information. Since the dynamics of carriers generated in the detector are determined by the presence of traps and defects, this technique is not just a method to characterize diamond nuclear detectors, rather it is a powerful tool that can be usefully applied to any kind of CVD diamond film to extract information on the identity of defects existing in CVD diamond.

\section{EXPERIMENT}

Several detector grade CVD diamond films have been deposited on silicon substrates ${ }^{13,14}$ in a microwave tubular reactor, suitably modified to improve film quality. ${ }^{15}$ A conventional scratching procedure was adopted in order to promote diamond nucleation on $\mathrm{Si}$ surface. The gas mixture used is $1 \% \mathrm{CH}_{4}$ in $\mathrm{H}_{2}$, resulting in a growth rate of about $0.7 \mu \mathrm{m} / \mathrm{h}$ at the deposition temperature of $750{ }^{\circ} \mathrm{C}$. Film thickness varied from about $20 \mu \mathrm{m}$ to about $100 \mu \mathrm{m}$, depending on the deposition time, and was measured with a digital thickness meter. The films showed excellent crystal quality, with very narrow diamond Raman peaks (FWHM about $2.4 \mathrm{~cm}^{-1}$ ) and extremely low photoluminescence background. ${ }^{15}$

Particle detectors were then realized by thermally evaporating a circular Au contact about $5 \mathrm{~mm}^{2}$ in size and $100 \mathrm{~nm}$ thick as the top electrode, while Ag paste was used as the silicon backing contact. The detector response to $5.5 \mathrm{MeV} \alpha$ particles, having a penetration depth of about $15 \mu \mathrm{m}$ in diamond, was studied using ${ }^{241} \mathrm{Am}$ source. The irradiation was carried out through a pinhole, in the normal direction to the sample surface. To measure the charge collection spectra the detector output has been connected, through a charge preamplifier and a shaping amplifier with a $2 \mu$ s shaping time, to a multichannel analyzer.

The charge collection spectra of the detectors were measured both in the as-grown state and after preirradiation with ${ }^{90} \mathrm{Sr} \beta$ particles for about $60 \mathrm{~h}$ (3 krad total dose), necessary to drive the film in a fully pumped state. A typical charge collection spectrum of our samples is reported in Fig. 1. This particular film is $100 \mu \mathrm{m}$ thick, as coming from a deposition time of about $150 \mathrm{~h}$, is in the pumped state, and the applied voltage is $100 \mathrm{~V}$. The average efficiency is about $40 \%$ (to be compared to the as-grown value of $9 \%$ ) corresponding to an average CCD of about $85 \mu \mathrm{m}$. Maximum values are about $65 \%$ and $185 \mu \mathrm{m}$, respectively. These high values reflect the 
high film quality. In all cases the whole film has been utilized to build the detector, i.e., without removing the low quality layer close to the substrate interface, a procedure normally used to increase the detector performance (see, e.g., Ref. 1). Since $\alpha$ particles are irradiated on the growth surface and their penetration depth is about $15 \mu \mathrm{m}$, this does not significantly reduce efficiency in thick films.

All the pulse time shapes have been measured on our samples using a fast digital oscilloscope, and have been corrected for the $3.2 \mu$ s decay time of the electronics as discussed below.

\section{TRAPPING-DETRAPPING MODEL}

Measuring the shape of the transient response of CdTe particle detectors Zanio et al. ${ }^{11}$ found that it is influenced by the presence of carriers trapping and release (detrapping) by defects. On this basis Martini and McMath ${ }^{12}$ quantitatively analyzed the behavior of $\mathrm{Ge}(\mathrm{Li})$ and $\mathrm{Si}(\mathrm{Li})$ detectors developing a model involving a single carrier type generated at one electrode and a single trapping center. In this case a single charge moving with drift velocity $v$ from one electrode to the other one in a parallel plate detector of thickness $d$ contributes by $d q=(e v / d) d t$ to the total charge induced in the external circuit as a result of Ramo's theorem. Introducing the transit time $T_{R}=d / v$, i.e., the time necessary for a carrier to traverse the detector in the absence of trapping processes, for $t<T_{R}$ no charge reaches the electrode and the rate equation for free carriers is

$$
\frac{d n}{d t}=-\frac{n}{\tau^{+}}+\frac{\left(n_{0}-n\right)}{\tau^{D}},
$$

where $n_{0}$ is the number of carriers generated by the ionizing particle, $\tau^{+}$is the trapping (i.e., free drift) time constant, and ${ }^{D}$ is the detrapping time constant. By using Ramo's theorem, Eq. (3) leads to

$$
Q(t)=\frac{Q_{0} \tau_{e}}{T_{R}}\left[\frac{t}{\tau^{D}}+\frac{\tau_{e}}{\tau^{+}}\left(1-e^{-t / \tau_{e}}\right)\right],
$$

with $Q_{0}$ the total charge generated by the ionizing particle at the electrode and $\tau_{e}=\tau^{+} \tau^{D} /\left(\tau^{+}+\tau^{D}\right)$. This formula holds for $t<T_{R}$, since afterwards some charges reach the electrode and the total number of carriers in the detector changes. As shown by Eq. (4), the time evolution of the pulses generated by an ionizing particle in a parallel plate detector depends on the defects present in the detector. In the absence of trapping defects the pulse rises linearly with time up to the transit time $T_{R}$ at which all charges generated are collected at the electrodes [Eq. (4) with $\tau^{+} \rightarrow \infty$ ]. In this case the detector's efficiency is $100 \%$. Traps reduce the efficiency (i.e., the pulse amplitude) if they do not allow thermal detrapping; otherwise $100 \%$ efficiency is still achieved, but the time response is now slower. Carriers experiencing trappingdetrapping have their motion delayed by a time of the order of the detrapping time constant, so that the charge collection process extends beyond the transit time $T_{R}$ i.e., outside the region of validity of Eq. (4). For a $100 \mu \mathrm{m}$ thick diamond detector working under an external field $E=10^{4} \mathrm{~V} / \mathrm{cm}, T_{R}$

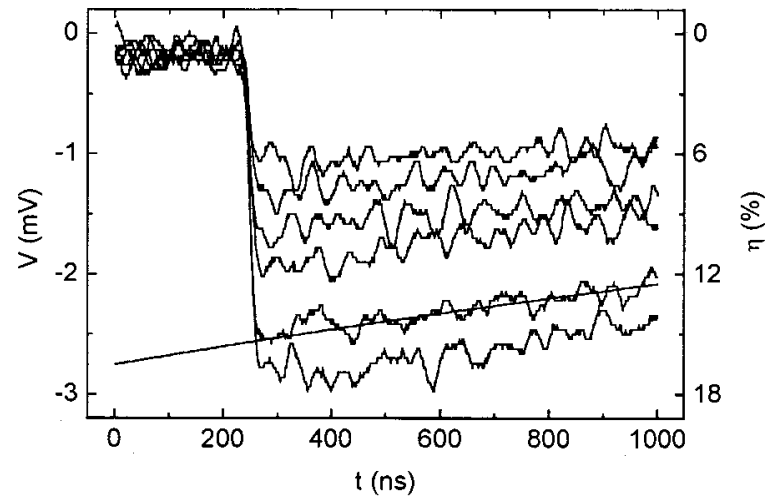

FIG. 2. Time evolution of the pulses measured in the as-grown state on the same film whose spectrum is reported in Fig. 1. The applied electric field is $10 \mathrm{kV} / \mathrm{cm}$, with negative polarity. The continuous line is a fit of one of the pulses by an exponential with a 3.2 $\mu$ s time constant due to the electronics.

$=d / v \approx d / \mu E \approx 1 \mathrm{~ns}$, so that in most cases the transit time in diamond detectors is shorter or comparable to the response time of the instrument used to trace pulse shapes, and the "fast" component described by Eq. (4) may not be experimentally accessible. Charge collection for $t>T_{R}$ (the socalled "slow" component of the pulse) is ruled by the detrapping time constant which can be much longer than a few nanoseconds, so that it can be experimentally measured.

Unfortunately the time behavior of this "slow" component is not accessible analytically even in this simple case involving a single type of trapping center and a single type of carrier generated at one electrode. Moreover, such a simple model cannot be applied to diamond detectors under $\alpha$ - or $\beta$-particle irradiation. In this case the released charge $Q_{0}$ is not concentrated at one electrode but distributed along the particle penetration depth, ranging from several microns to the whole sample thickness; consequently both electrons and holes can in principle contribute to charge collection. In addition, we will show in the following that more than a single type of defect must be introduced to explain experimental results in the case of CVD diamond detectors. A computer simulation can be used anyway to quantitatively describe trapping-detrapping effects once a qualitative picture of the trapping centers present in the detectors is available. The following section describes this procedure.

\section{RESULTS AND DISCUSSION}

\section{A. Qualitative analysis}

We now examine the experimental time evolution of the pulses before pumping (i.e., in the as-grown state). Let us consider the same film whose spectrum in the pumped state is reported in Fig. 1. The pulses are plotted in Fig. 2 for the sample in the as-grown state under negative field polarity (the behavior for positive field polarity is very similar; see below). The applied voltage is $100 \mathrm{~V}$, corresponding to an electric field of $10 \mathrm{kV} / \mathrm{cm}$. After the initial fast rise, a slow decrease is found for all pulses. In all cases, this decrease is very well fitted by an exponential with a $3.2 \mu$ s time constant and it is therefore due to the electronics. In the following, all 


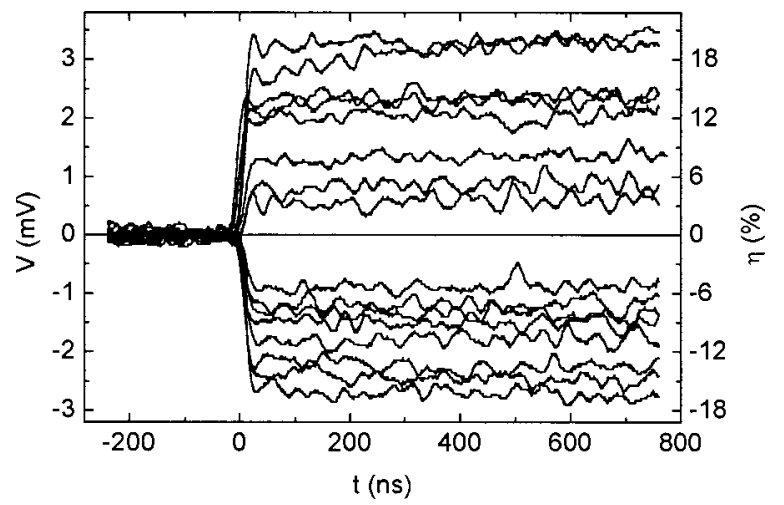

FIG. 3. Pulses measured in the as-grown state for both positive and negative field polarity after correction for the electronics decay time.

pulses are corrected for this decay, so that their time behavior represents the actual detector response. Replotting the pulses measured in the as-grown state (for both positive and negative field polarity) after correction we obtain Fig. 3. It is evident that the pulses have different amplitudes, reflecting the width of the charge collection spectrum. However, similar amplitudes are observed for positive and negative polarity. The most important feature is that in all cases the saturation value is immediately reached, the collected charge being constant after the $10 \mathrm{~ns}$ rise time of the digital oscilloscope. This implies that once trapped by a defect, electrons and holes are not detrapped. Since thermally activated detrapping effects do not significantly contribute to charge collection, the defects limiting the detector's response in the as-grown state are identified as deep ones. The density of these trapping centers is such that the CCD is about $12 \mu \mathrm{m}^{5}$. No significant difference is observed between positive and negative polarity because CCD (and even more so both electron and hole mean free paths before trapping) is lower than the penetration depth $G \cong 15 \mu \mathrm{m}$ of $5.5 \mathrm{MeV} \alpha$ particles in diamond, so that the growth surface boundary plays a very limited role. Further, since the film thickness is $d \gg \delta$ the substrate interface boundary plays no role at all. Therefore, even if electrons and holes should have different mean free paths, no difference would be observed between negative and positive polarity. In principle only one kind of trap for electrons and holes is necessary to explain the behavior in the as-grown state.

This picture changes when examining the pulse shapes for the same sample, but in the pumped state, which are plotted in Fig. 4. No significant change occurs in the case of negative polarity with respect to the unpumped state. A dramatic change, on the other hand, can be seen for positive field polarity. Not only is the amplitude of the pulses greatly enhanced, reflecting the large increase of efficiency due to pumping, but a significant slow component develops. The symmetry between positive and negative polarity is therefore now broken.

To explain this behavior it is necessary to assume that another kind of defect becomes important in the charge collection process after pumping, namely a relatively shallow trap for holes, allowing detrapping. It seems that pumping

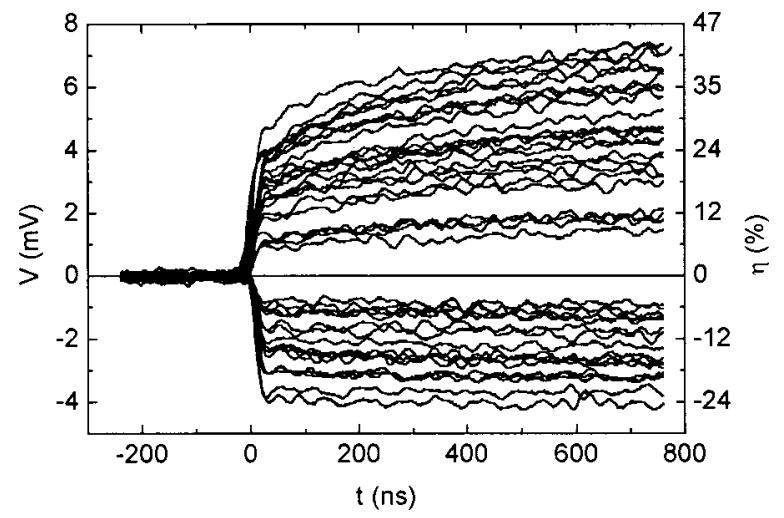

FIG. 4. Pulses measured in the pumped state for both positive and negative field polarity after correction for the electronics decay time.

leads to a much higher mean free path for holes than in the as grown state, while the electron mean free path is not strongly affected. Thus, in the case of positive polarity holes are now substantially free to move along the $100 \mu \mathrm{m}$ film thickness towards the substrate interface, while for negative polarity they are collected at the upper electrode after having traveled at most a distance $G \cong 15 \mu \mathrm{m}$.

The simplest picture compatible with all experimental facts, including the development of the slow component, is therefore as follows. In the pumped state the mean free path of holes is greatly enhanced, because pumping saturates most of the deep defects responsible for hole trapping without detrapping. Thus, shallower traps existing in a lower concentration than saturated, deeper ones, now become important. Since they allow detrapping, they do not limit the overall amplitude of the pulse, but slow down the process of charge collection. The main features of Fig. 4 are due to this effect. For positive polarity holes are now substantially free to travel through most of the sample via trapping-detrapping processes, thus leading to high collection efficiency ${ }^{4}$ and to the development of the slow component. For negative polarity, the limited changes in the pulse amplitude and shape with respect to the as-grown state show that no significant saturation of electron traps occurs. Holes are in principle substantially free to move, but since $5.5 \mathrm{MeV} \alpha$ particles ionize only within their penetration depth $G \simeq 15 \mu \mathrm{m}$ from the detectors' surface (much less than the hole mean free path, see Table I), holes can move only a few microns before being collected at the upper electrode. They therefore cannot give a great contribution to charge collection according to Ramo's theorem, nor can be trapped and detrapped since their mean free path is now much higher than the real path to the electrode. Therefore the slow component substantially does not show up, and at the same time the fast one does not increase significantly with respect to the as-grown case.

In conclusion, qualitative analysis of pulse shapes show that both deep (type $A$ ) and shallow (type $B$ ) defects exist for holes, while only deep (type $A$ ) defects are present for electrons. Note that, of course, $A / B$ here simply stand for deep/ shallow, and by no means are $A$ defects for holes physically identified with $A$ defects for electrons. 


\section{B. Computer simulation}

Since the trapping-detrapping model seems to qualitatively explain our results, we developed a computer simulation according to it, allowing us to calculate, in a discrete time approximation, the pulse shape $Q(t)$. For each carrier type, the detector of thickness $d$ is divided into $N$ laminae, the transit time of a carrier in each lamina being $\Delta t=d / N v$ $=T_{R} / N$, where $v$ is the drift velocity. For each type of carrier (electrons and holes) two kinds of defects $(\mathrm{A}, \mathrm{B})$ are taken into account (see above), having trapping time constants $\tau_{A}^{+}, \tau_{B}^{+}$and detrapping time constants $\tau_{A}^{D}, \tau_{B}^{D}$, respectively. Carriers can be free, $n_{i}\left(t_{j}\right)$ being the number of free carrier in the $i$ th lamina at time $t_{j}$, or trapped in each of the two trapping centers, $n_{A i}\left(t_{i}\right), n_{B i}\left(t_{i}\right)$ being their corresponding numbers. In the time interval $\Delta t=t_{j}-t_{j-1}$, free carriers either move to the next layer contributing by $e v \Delta t / d=e / N$ to the induced charge, or are trapped in either defect. In the same time interval, trapped carriers can be released and move to the next layer or remain trapped. We have therefore at first order approximation in $\Delta t$ :

$$
\begin{aligned}
n_{i}\left(t_{j}\right)= & n_{i-1}\left(t_{j-1}\right)-n_{i-1}\left(t_{j-1}\right) \Delta t\left(\frac{1}{\tau_{A}^{+}}+\frac{1}{\tau_{B}^{+}}\right) \\
& +n_{A(i-1)}\left(t_{j-1}\right) \frac{\Delta t}{\tau_{A}^{D}}+n_{B(i-1)}\left(t_{j-1}\right) \frac{\Delta t}{\tau_{B}^{D}}, \\
n_{A i}\left(t_{j}\right)= & n_{A i}\left(t_{j-1}\right)-n_{A i}\left(t_{j-1}\right) \frac{\Delta t}{\tau_{A}^{D}}+n_{i}\left(t_{j-1}\right) \frac{\Delta t}{\tau_{A}^{+}}, \\
n_{B i}\left(t_{j}\right)= & n_{B i}\left(t_{j-1}\right)-n_{B i}\left(t_{j-1}\right) \frac{\Delta t}{\tau_{B}^{D}}+n_{i}\left(t_{j-1}\right) \frac{\Delta t}{\tau_{B}^{+}}
\end{aligned}
$$

(release and trapping of a carrier in a single time interval $\Delta t$ and other second order effects can be neglected for sufficiently large $N$ values). The total charge induced by each type of carrier is then

$$
Q\left(t_{j}\right)=Q\left(t_{j-1}\right)+\frac{e}{N} \sum_{i} n_{i}\left(t_{i}\right) .
$$

This procedure is repeated twice (for electrons and holes, respectively), and the results are summed. In Eqs. (5)-(7) direct recombination effects have been neglected since electrons and holes rapidly separate under the external electric field, and the concentration of charges generated along the $\alpha$-particle track is estimated to be lower than that of trapping centers. Equations (5)-(7) are the discreet counterparts of the differential equations

$$
\begin{gathered}
\frac{\partial n(x, t)}{\partial t}=-v \frac{\partial n(x, t)}{\partial x}-\frac{\partial n_{A}(x, t)}{\partial t}-\frac{\partial n_{B}(x, t)}{\partial t}, \\
\frac{\partial n_{A}(x, t)}{\partial t}=\frac{n(x, t)}{\tau_{A}^{+}}-\frac{n_{A}(x, t)}{\tau_{A}^{D}},
\end{gathered}
$$

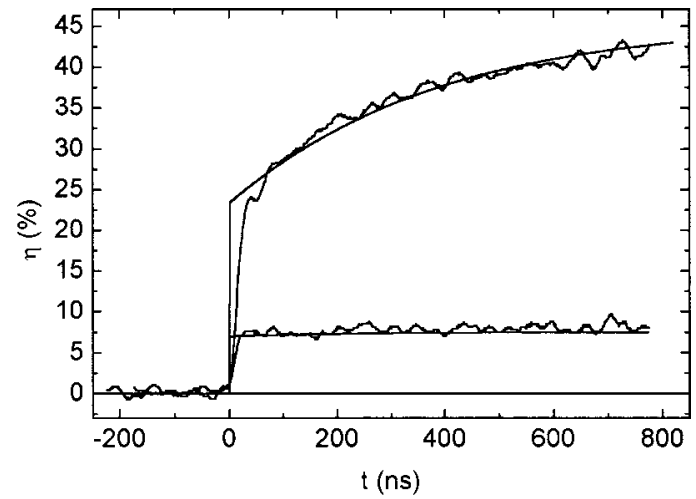

FIG. 5. Comparison of measured pulses with the simulation based on the trapping-detrapping model.

$$
\frac{\partial n_{B}(x, t)}{\partial t}=\frac{n(x, t)}{\tau_{B}^{+}}-\frac{n_{B}(x, t)}{\tau_{B}^{D}},
$$

describing the charge densities $n(x, t), n_{A}(x, t)$, and $n_{B}(x, t)$. The model can be easily extended to include other types of defects.

In the general case, the parameters to be included in the simulation are the carrier velocity (which gives the value of $\Delta t$ ), and the time constants $\tau_{e A}^{+}, \tau_{e B}^{+}, \tau_{e A}^{D}, \tau_{e B}^{D}$ and $\tau_{h A}^{+}$, $\tau_{h B}^{+}, \tau_{h A}^{D}, \tau_{h B}^{D}$ for electrons and holes, respectively. However, in our case, according to the discussion reported in the previous section, the density of type $B$ defects for electrons is taken to be zero (i.e., $\left.\tau_{e B}^{+}=\infty\right)$. Also, type $A$ defects are deep ones and do not allow detrapping $\left(\tau_{e A}^{D}, \tau_{h A}^{D}=\infty\right)$. Only $\tau_{e A}^{+}$, $\tau_{h A}^{+}$and $\tau_{e B}^{+}, \tau_{h B}^{D}$ must therefore be determined. Since the CCD is more easily visualized in terms of carrier mean free path, and trapping times are directly connected to mean free paths through the carrier velocity $v$, the parameters which we will discuss are the mean free paths $L_{e A}=v \tau_{e A}^{+}, L_{h A}$ $=v \tau_{h A}^{+}, L_{h B}=v \tau_{h B}^{+}$due to type $A$ or $B$ defects for electrons and holes, respectively, and the detrapping time constant for holes, $\tau_{h B}^{D}$. In the simulation, the carrier velocity has been fixed to $510^{6} \mathrm{~cm} / \mathrm{s}$. ${ }^{16}$ The total transit time of a carrier through the detector is therefore $T_{R}=d / v=2 \mathrm{~ns}$, and all time constant values given are relative to this value, while the mean free path values do not depend on $v$ and are therefore real ones.

In Fig. 5 simulated pulses are compared with experimental ones in the as-grown and pumped states for positive polarity (the negative polarity case is a trivial one, since the slow component is always very small). Because of the scaling property of the pulses described below, a single pulse for the as-grown and pumped states has been selected, chosen in the middle of the efficiency distribution determined by the $\alpha$-particle collection spectra (i.e., about $8 \%$ in the as-grown state and about $40 \%$ in the pumped state).

The simulation describes very well the experimental behavior of both curves using a single set of physical parameters, whose values are reported in Table I. The procedure adopted to find the parameters values giving the best agreement with the measured pulses is as follows. First, the asgrown curve alone is simulated. Since, as discussed above, 
TABLE I. Fitting parameters used in the simulation of Fig. 5. $L_{e A}, L_{h A}$ are the mean free paths due to type $A$ defects for electrons and holes, respectively, $L_{h B}$ is the mean free path for holes due to type $B$ defects, and $\tau_{h B}^{D}$ is the detrapping time constant for holes.

\begin{tabular}{ccc}
\hline \hline & Before pumping & After pumping \\
\hline$L_{e A}(\mu \mathrm{m})$ & 4.5 & 4.5 \\
$L_{h A}(\mu \mathrm{m})$ & 4.5 & 50 \\
$L_{h B}(\mu \mathrm{m})$ & 35 & 35 \\
$\tau_{h B}^{D}(\mathrm{~ns})$ & 200 & 200 \\
\hline \hline
\end{tabular}

the lack of a slow component for the as-grown detector in positive polarity means that the density of deep $(A)$ traps for holes is much higher than that of shallow $(B)$ traps, the only parameters to be determined are in this case $L_{e A}$ and $L_{h A}$, the value of $L_{h B}$ being almost irrelevant. When, as in the present case, the efficiency is so low that the total CCD is lower than the penetration depth of $\alpha$ particles, the only accessible information is the sum of electron and hole mean free paths and not their separate values. Therefore we set $L_{e A}=L_{h A}$ and found that the as-grown state pulse is simulated using $L_{e A}=L_{h A} \cong 4.5 \mu \mathrm{m}$ for a total CCD of about 9 $\mu \mathrm{m}$. This agrees well with the $12 \mu \mathrm{m}$ value reported in Ref. 5 for samples thicker than $40 \mu \mathrm{m}$, taking into account the fact that in the present work the applied field is $10 \mathrm{kV} / \mathrm{cm}$, lower than the one used in Ref. $5(15 \mathrm{kV} / \mathrm{cm})$. With the chosen value for the carrier velocity, these mean free paths correspond to $\tau_{e A}^{+}=\tau_{h A}^{+}=0.09 \mathrm{~ns}$. Hence the pulse measured in the pumped state has been simulated introducing also shallow (detrapping) hole traps (i.e., $L_{h B}$ ) and, as already argued in the preceding section, a substantially lower concentration of effective deep hole traps (i.e., a higher value for $L_{h A}$ ) as a consequence of pumping. The concentrations of deep electron traps (i.e., $L_{e A}$ ) is kept unaltered. In order to reproduce the experimentally observed amplitude ratio between fast and slow components it turns out that $L_{h A}=50 \mu \mathrm{m}$ (i.e., $\tau_{h A}^{+}=1 \mathrm{~ns}$ ), so that the pumping process reduces the density of effective hole deep trapping centers by one order of magnitude, while $L_{h B}=35 \mu \mathrm{m}$ (i.e., $\tau_{h B}^{+}=0.7 \mathrm{~ns}$ ), much higher than the $4.5 \mu \mathrm{m}$ as-grown value of $L_{h A}$. This is consistent with our assumption that before pumping deep (type $A$ ) traps dominate hole dynamics (coming from the lack of a significant slow component); thus the validity is confirmed of the initial assignment $L_{e A}=L_{h A} \cong 4.5 \mu \mathrm{m}$ based upon neglecting hole type $B$ traps in the simulation of the as-grown pulse.

The time evolution of the slow component is well described when $\tau_{h B}^{D}=200 \mathrm{~ns}$. From the $200 \mathrm{~ns}$ detrapping time a rough estimate of the activation energy $E_{D}$ of shallow defects responsible for hole trapping and detrapping can be made since $1 / \tau_{h B}^{D}=s \exp \left(-E_{D} / k T\right)$. Using $s \approx 10^{12} \mathrm{~s}^{-1}$ for the attempt frequency (to which $E_{D}$ is anyway relatively insensitive) we obtain $E_{D} \cong 0.3 \mathrm{eV}$. Finally, all parameters are introduced in the simulation and both curves are recalculated refining all parameters together, and the result is plotted in Fig. 5.

In agreement with the model, in much thinner films ( $d$ $\leqslant 35 \mu \mathrm{m}$ ) a significant slow component is not detected even after pumping, since holes are trapped at the highly defective region close to the film-substrate interface before traveling distances much in excess of $10 \mu \mathrm{m}$.

\section{Field effect}

A very interesting feature of the pulses reported in Figs. 3 and 4 is the similarity of their shapes, in spite of the large differences in their amplitude. We therefore normalized them, and found that within the experimental noise, the normalized pulses superimpose well, except for small deviations in the region of the initial rise of the slow component. This scaling is rather obvious when only the fast component is present, but certainly not trivial in the case of the pulses measured for positive polarity on the detector in the pumped state, for which a consistent fraction of the collected charge comes from the slow component. This means that for all the reported pulses (which span an efficiency "window" roughly extending from $15 \%$ to $50 \%$ ) the ratio between the fast and the slow component is very similar. This scaling would point in favor of a single, simple mechanism affecting the pulse amplitudes. It would have been tempting to attribute the different pulse amplitudes, and therefore the broadening of the charge collection spectra, to electric field inhomogeneities due to the polycrystalline nature of CVD diamond.

To better clarify the role of the electric field we report in Fig. 6 normalized pulses measured at various applied voltages from $30 \mathrm{~V}$ to $180 \mathrm{~V}$ (corresponding to $3 \mathrm{kV} / \mathrm{cm}$ and 18 $\mathrm{kV} / \mathrm{cm}$, respectively). Pulses are grouped according to the applied voltage ( 3 pulses for each voltage, from low to high efficiency) and different groups are vertically shifted to increase readability. It can be seen that within each group pulses scale well, with the exception of a single pulse at 60 $\mathrm{V}$, but as the voltage is increased the time behavior of the slow component speeds up. Two possible explanations are (a) field enhanced detrapping, and (b) the occurrence of multiple trapping-detrapping events which slow down the response at lower fields, when the transit time $T_{R}$ is increased. In the latter case, the simulation shows that to account for the observed speed up, the transit time $T_{R}$ should change by a factor comparable to the sixfold increase in field, so that the ratio between trapping times and $T_{R}$ sweeps from above 1 to significantly below 1 (assuming that trapping times are not affected in the same way by carrier drift velocity, i.e., the cross section decreases with the carrier drift velocity $v$ ). This seems unlikely since at fields of the order of about $10^{4} \mathrm{~V} / \mathrm{cm}$ (i.e., of the order of the ones used by us) the carrier drift velocity of natural diamond is close to saturation. Moreover, the substantial constancy of the ratio between the slow and fast components which appears from Fig. 6 seems to rule out this possibility as the main source of pulse speedup.

Indeed, in the approximate case when the ionized charge is generated at one electrode, and the detrapping time constant is much longer than the trapping time constant as it is in our case, the ratio between the slow and fast components can easily be analytically calculated. In this case, the fast component is clearly given by Eq. (4) with $t=T_{R}$ and $\tau^{D}=\infty$, i.e., by $h\left(\tau_{\|}^{+} / T_{R}\right)$, where $\tau_{\|}^{+}=\tau_{A}^{+} \tau_{B}^{+} /\left(\tau_{A}^{+}+\tau_{B}^{+}\right)$and $h(x)$ 


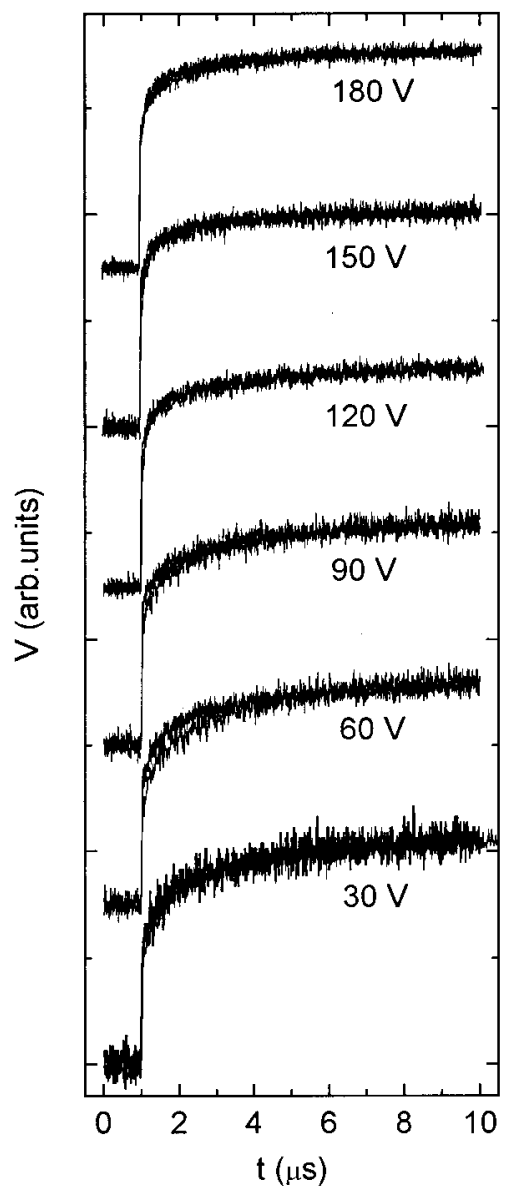

FIG. 6. Normalized pulses grouped according to the voltage applied during the measurement (from $30 \mathrm{~V}$ to $180 \mathrm{~V}$ in $30 \mathrm{~V}$ increments).

$=x(1-\exp (-1 / x))$ is the Hecht function of argument $x$. On the other hand, the total efficiency is given by the same formula in which detrapping centers, which do not lead to loss of signal, are removed (i.e., $\tau_{\|}^{+}=\tau_{A}^{+}$) and therefore by $h\left(\tau_{A}^{+} / T_{R}\right)$. The ratio $R$ between the slow and the fast component is then given for a single carrier by

$$
R=\frac{h\left(\tau_{A}^{+} / T_{R}\right)}{h\left(\tau_{\|}^{+} / T_{R}\right)}-1,
$$

so that $R$ only depends on the ratio between trapping times and $T_{R}$.
According to Eq. (9), and using the parameter values found in Sec. IV B, when $T_{R}$ is varied by a factor of 6 from $0.7 \mathrm{~ns}$ to $4 \mathrm{~ns}, R$ should change from 1.21 to 0.34 , while the total efficiency increases from $17 \%$ to $47 \%$. Although the model does not take into account all the features of polycrystalline diamond, according to Fig. 6 it would seem that multiple trapping-detrapping effects should not be the main source of the pulse speedup. Also, higher fields do not lead to a marked change in the slow to fast component ratio since, in the range of fields explored by us, the carrier drift velocity is close to saturation so that $T_{R}$ is not strongly affected by the field. Field-assisted detrapping is therefore likely to occur at the highest fields. Although $E_{D}$ is relatively high in our case, field-assisted detrapping has been observed in CdS (Ref. 17) and $\mathrm{Se}$ (Ref. 18) $\left(E_{D} \cong 0.1 \mathrm{eV}\right)$, and the speedup effect shown in Fig. 6 is not dramatic given the sixfold increase in field.

Qualitatively similar results have been obtained on other samples, so that the described behavior can be considered as a general property of our high quality CVD diamond films.

\section{CONCLUSIONS}

An analysis of the time pulse shape of particle detectors built from high quality CVD diamond films has been carried out. It is found that the pulse amplitude and shape depend on the field polarity and is dramatically affected by pumping. These changes are interpreted in the framework of the trapping-detrapping model originally applied to Si-based detectors. ${ }^{12}$ To explain the particular features found in this work the original model has to be modified to reflect the higher complexity of CVD diamond. A computer simulation based on the model gives a pulse shape which agrees well with the observed ones. Valuable information concerning the nature of trapping centers for electrons and holes is provided by the analysis of pulse shapes. In particular, at least two different trapping centers for holes are identified, the shallower of the two having activation energy close to $0.3 \mathrm{eV}$.

The effect of pumping, which results in an increased amplitude and in the development of a slow component for positive field polarity, is explained, allowing us to enlighten the role of pumping in the detector's performance and the different behavior of electrons and holes. A remarkable feature is the apparent scaling (within the experimental noise) of pulses measured in a given condition, which all have the same shape, independently from their amplitude. The behavior of the response under increasing electric fields leads to the conclusion that field enhanced detrapping occurs at fields close to $10^{4} \mathrm{~V} / \mathrm{cm}$.
${ }^{1} \mathrm{~W}$. Adam et al., in 5th International Symposium on Diamond Materials, Paris, France, 1997, edited by J. L. Davidson et al., Proceedings of the Electrochemical Society Vol. 97-32 (Electrochemical Society, Pennington, NJ, 1998), p. 491.

${ }^{2}$ D. R. Kania, in The Physics of Diamond, edited by A. Paoletti and A. Tucciarone (IOS Press, Amsterdam, 1997).
${ }^{3}$ Y. Tanimura, J. Kaneko, M. Katagiri, Y. Ikeda, T. Nishitani, H. Takeuchi, and T. Iida, Nucl. Instrum. Methods Phys. Res. A 443, 325 (2000).

${ }^{4}$ M. Marinelli, E. Milani, A. Paoletti, A. Tucciarone, G. Verona Rinati, M. Angelone, and M. Pillon, Appl. Phys. Lett. 75, 3216 (1999). 
${ }^{5}$ M. Marinelli, E. Milani, A. Paoletti, A. Tucciarone, G. Verona Rinati, M. Angelone, and M. Pillon, J. Appl. Phys. 89, 1430 (2001).

${ }^{6}$ C. Manfredotti, F. Fizzotti, A. LoGiudice, P. Polesello, E. Vittone, R. Lu, and M. Jaksic, Diamond Relat. Mater. 8, 1597 (1999).

${ }^{7}$ W. Shockley, J. Appl. Phys. 9, 635 (1938).

${ }^{8}$ S. Ramo, Proc. IRE 27, 584 (1939).

${ }^{9}$ C. Manfredotti, F. Fizzotti, A. LoGiudice, P. Polesello, E. Vittone, M. Truccato, and P. Rossi, Diamond Relat. Mater. 8, 1592 (1999).

${ }^{10}$ D. R. Beckman, A. Saint, P. Gonon, D. N. Jamieson, S. Prawer, R. Kalish, Nucl. Instrum. Methods Phys. Res. B 130, 518 (1997).

${ }^{11}$ K. Zanio, W. Akutagawa, and J. W. Mayer, Appl. Phys. Lett. 11, 5 (1967).

${ }^{12}$ M. Martini and T. A. McMath, Nucl. Instrum. Methods 79, 259
(1970).

${ }^{13}$ G. Balestrino, M. Marinelli, E. Milani, A. Paoletti, I. Pinter, and A. Tebano, Appl. Phys. Lett. 62, 879 (1993).

${ }^{14}$ M. Marinelli, E. Milani, M. Montuori, A. Paoletti, P. Paroli, and J. Thomas, Appl. Phys. Lett. 65, 2839 (1994).

${ }^{15}$ M. Marinelli, E. Milani, A. Paoletti, A. Tucciarone, G. Verona Rinati, G. Messina, M. Angelone, and M. Pillon, Proceedings of the Applied Diamond Conference/Frontier Carbon Technology Joint Conference, Tsukuba, Japan (AIST, Tsukuba, Japan, 1999), p. 154.

${ }^{16}$ S. Zhao, Ph.D. dissertation, Ohio State University, 1994.

${ }^{17}$ K. V. Boer and V. K. Kummel, Ann. Phys. (Leipzig) 14, 341 (1954).

${ }^{18}$ M. D. Tabak and P. J. Warter, Phys. Rev. 173, 899 (1968). 